\title{
Comparative Proteomic Analysis of Chicken Primary Hepatocytes with Folic Acid Free or Supplementation Culture Mediums
}

\author{
Yanli Liu, Jianfei Zhao, Fangyuan Wang, Jinghui Zhou, Xin Yang and Xiaojun Yang* \\ College of Animal Science and Technology, Northwest A\&F University, Yangling, China \\ *Corresponding author’s Email: yangxj@ nwsuaf.edu.cn; ORCID: 0000-0001-9702-7039
}

Received: 17 Jan. 2020

Accepted: 19 Feb. 2020

\begin{abstract}
Folic acid had been reported to develop much metabolic regulation function in animals and human beings due to its roles in one carbon metabolism. The current study was conducted to explore folic acid regulation function in primary chicken hepatocytes via supplement and deprivation culture models based on proteomic analysis. Results have shown that folic acid supplement significantly increased intracellular folic acid, 5-Me-THF and SAM contents when compared with folic acid free group $(\mathrm{P}<0.05)$. Whereas, there was no difference about genome $5 \mathrm{mC}$ levels and DNMTs mRNA expression between these two groups. Proteomic analysis found 85 differential expressed proteins with 35 down and 50 up regulation. COG and KEGG pathway analysis revealed that amino acid metabolism, carbohydrate metabolism and antioxidant function were affected by folic acid. Posttranslational modification, protein turnover, chaperones and transcription were gathered by COG analysis in relative high proportion. PRMT7 and ARID4B which were associated with histone methylation were up-regulated in the folic acid supplement group, suggesting that folic acid was likely to take part in metabolism regulation of hepatocytes via histone methylation manner in the study. In conclusion, proteomic analysis found 85 differential expressed proteins in hepatocytes with folic acid free and supplementation medium. Folic acid might be involved in amino acid and carbohydrate metabolism and oxidation resistance by its epigenetic modifications functions. Our study also provided fundamental differential protein profiles mediated by folic acid, which can facilitate the understanding of folic acid regulation function in hepatic metabolism.
\end{abstract}

Key words: Folic acid, Histone methylation, Primary chicken hepatocytes, Proteomics

Abbreviations: MTHFR: methylenetetrahydrofolate reductase; FA: folic acid; DNMT: DNA methyltransferase; GO: Gene ontology; COG: cluster of orthologous groups of proteins; DEP: differential expressed protein; ROS: Reactive oxygen species; KEGG: Kyoto Encyclopedia of Genes and Genomes.

\section{INTRODUCTION}

Folic acid, as an essential B vitamin, had been reported to develop many metabolic regulation functions in animals and human beings. For instance, folic acid addition could reduce hypoxia-induced inflammatory response by Reactive oxygen species and JAK2/STAT3 pathway in human pro-myelomonocytic cells (Ma et al., 2018), and also could anises acetate-induced hepatotoxicity by downregulating NF- $\kappa B$, IL-1 $\beta$ production and lipid peroxidation caused by cell injury (Allah and Badary, 2017). What's more, maternal use of folic acid can prevent many neural tube defects (Molloy et al., 2017). The previous study also revealed that folic acid decreased homocysteine level and improved antioxidative capacity in atherosclerotic rats (Cui et al., 2017). In addition, folate was reported to have prevention function in breast cancer risk (Chen et al., 2014). On the other hand, many study reported that folic acid developed function by changing DNA methylation because of its roles in one-carbon transfer reactions; Yu et al. (2014) has found that folic acid could reduce lipid accumulation of chicken adipocytes by increasing DNA methylation of $\mathrm{C} / \mathrm{EBP} \alpha$ promoter, thereby reducing FAS and PPAR $\gamma$ expression. It was reported that the mouse sperm epigenome would be altered under the condition of low paternal dietary folate (histone $\mathrm{H} 3$ methylation or DNA methylation), which was also associated with many negative pregnancy outcomes (Lambrot et al., 2013). Therefore, it's confirmed to some extent that folic acid could have anti-inflammation and anti-oxidation effect, and also play positive roles in some diseases. 
The liver is a metabolic organ owning synthesis, transportation, detoxication functions and also a major place of folic acid metabolism. Folic acid is transported inside the cell via different processes involving membrane embedded folate receptors or reduced folate carrier (Nazki, et al., 2014), then 5,10-methylenetetrahydrofolate could be distributed towards methionine pathways, which involves in remethylation of homocysteine for genomic and nongenomic methylation, catalyzed by methylenetetrahydrofolate reductase (MTHFR) through a non-reversible process (Lucock, 2000). In poultry industry, many metabolic diseases occur under the conditions of intensive breeding environment and higher improvement of growth performance by genetic breeding. It's aimed to come up with an assumption that whether folic acid could take part in hepatic metabolism regulation through DNA methylation capacity to solve the potential problems in chickens.

Hepatocytes culture in vitro is a suitable model to study metabolism, pharmacology and toxicology (Hou et al., 2001, Xu et al., 2012, Chen et al., 2017). And given the importance of liver organ itself in body metabolism and the metabolism site of folic acid, primary chicken hepatocytes will be used to explore our hypothesis mentioned above preliminarily in virtue of proteomics analysis technique. In addition, folic acid supplemented and folic acid deficient culture media are used to establish two cells culture models.

\section{MATERIALS AND METHODS}

\section{Culture of chicken primary hepatocytes}

Hepatocytes were isolated from male one-day-old layer chicks by collagenase digestion and filtration according to our previous description (Liu et al., 2018). We confirm that all animals' procedures used in the current study were approved by the ethical standards of the Animal Care and Use Committee of the College of Animal Science and Technology of the Northwest A\&F University (Shaanxi, China). After $12 \mathrm{~h}$ attachment incubation, hepatocytes were washed with PBS and replaced with growth medium; when the confluence reached to about $80 \%$, folic acid-free $(0 \mathrm{mg} / \mathrm{L})$ or folic acid supplemented medium $(15 \mathrm{mg} / \mathrm{L})$ was used to replace the normal medium $(1 \mathrm{mg} / \mathrm{L}$ folic acid) for another $12 \mathrm{~h}$ treatment. RPMI 1640 culture medium with folic acid-free was purchased from Gibco (Life Technologies, Carlsbad, CA) and folic acid from Sigma (St. Louis, MO). There are three replicates in each group for proteomics analysis, and six replicates for other detections. The folic acid was dissolved in the $10 \%$ ammonium hydroxide with minimal volume, then diluted to the concentration of $500 \mathrm{mg} / \mathrm{L}$ using deionized water (Yu et al., 2014), finally filtered by $0.22-\mu \mathrm{m}$ filters. The stock solution was diluted further in culture medium to reach the final concentrations required.

\section{$5 \mathrm{mC}$ level}

Genomic DNA from hepatocytes was isolated using the TIANamp Genomic DNA Kit (Tiangen, Beijing, China) according to standard procedures. Then $100 \mathrm{ng}$ of each DNA sample was used to measure global DNA methylation level using 5-mC DNA Elisa Kit (Zymo Research, Irvine, California, USA). The amount and percentage of methylated DNA $(5 \mathrm{mC})$ in the total DNA was calculated based on a standard curve.

\section{Determination of folic acid, 5-Me-THF and SAM contents}

Upon treatments, cells were rinsed with ice-cold PBS and trypsinized. Hepatocytes were centrifuged, washed and suspended in PBS. After ultrasonic decomposition, cells were centrifuged at $1500 \mathrm{~g}$ for $15 \mathrm{~min}$ at $4^{\circ} \mathrm{C}$ to remove cellular debris. The supernatant was collected to examine levels of folic acid, 5-Me-THF and SAM by Enzyme-linked Immunosorbent Assay Kits (Cloud-Clone Corp, USA). All the results were expressed as ng $/ 10^{6}$ cells.

\section{RNA isolation and gene quantification}

After the removal of treatment medium, cells were washed twice with ice-cold PBS. Total RNA was extracted based on the TRIZOL reagent instruction (Invitrogen, Carlsbad, CA). Its concentration and purity were determined by the absorbance at $260 \mathrm{~nm}$ and A260/A280 value using a NanoDrop 2000c spectrophotometer (Thermo Scientific, Wilmington, USA). $500 \mathrm{ng}$ of total RNA were used to complete cDNA synthesis by Primer Script RT Reagent Kit (TaKaRa, Dalian, China). Then the SYBR Premix Ex Taq kit (TaKaRa, Dalian, China) was used to carry out the assay for gene expression. Primers sequences were shown in table 1 . Detailed procedures were operated as our previous description (Liu, et al., 2016). The $2^{-\Delta \Delta C t}$ method was used for gene relative expression (Livak and Schmittgen, 2001).

\section{Protein extraction}

After treatment, cells were completely homogenized with a STD buffer (4\% SDS, $1 \mathrm{mM}$ DTT, $150 \mathrm{mM}$ Tris$\mathrm{HCl} \mathrm{pH} \mathrm{8.0,} \mathrm{protease} \mathrm{and} \mathrm{phosphatase} \mathrm{inhibitors),} \mathrm{then} \mathrm{the}$ mixture was heated at $100{ }^{\circ} \mathrm{C}$ for $10 \mathrm{~min}$. After centrifugation at $12000 \mathrm{~g}$ for $10 \mathrm{~min}$ when cooled to room 
temperature, the supernatants were collected and protein concentration was determined using the Bicinchoninic acid (BCA) assay kit (Bio-Rad) based on its protocols.

\section{Protein digestion and iTRAQ labelling}

A total of $200 \mu \mathrm{g}$ protein were digested following the reported methods (Du et al., 2015), and the peptide content was quantified by UV light spectral density at $280 \mathrm{~nm}$. Then $80 \mu \mathrm{g}$ peptide for each sample were used for iTRAQ labelling (Applied Biosystems). The three samples in 0 $\mathrm{mg} / \mathrm{L}$ group were labelled with reagents 113,114 and 115 . The samples in $15 \mathrm{mg} / \mathrm{L}$ group were labelled with 118 , 119 and 121. After labelling, all samples were pooled and dried. The mixed labeled peptides were carried out fractionating by strong cationic-exchange (SCX) chromatography separation. About 36 fractions were collected and combined, then desalted on C18 Cartridges. Each fraction was detected for liquid chromatographytandem mass spectrometry (LC-MS/MS). Detailed procedures are on the basis of previous report (Dong, et al., 2017, Cao, et al., 2018).

\section{Protein identification and quantification}

The protein identification and iTRAQ quantification were operated using a Mascot 2.2 (Matrix Science, London, UK) and Proteome Discoverer 1.4 (Thermo Electron, San Joes, CA) as described (Wang, et al., 2013). The corresponding parameters were set as same as the description by Du et al. (2015). Database search was performed against the Gallus (Uniprot) database. For statistical analysis, student's $t$ test was used to identify significant changes between two group samples. Proteins with a statistically significant iTRAQ ratio of $>1.2$ or < $0.83(\mathrm{P}<0.05)$ were considered differentially abundant proteins.

\section{Functional analysis}

Gene ontology (GO), cluster of orthologous groups of proteins (COG), KEGG pathways and proteins interaction of identified differential proteins were analyzed respectively according to previously reported method ( $\mathrm{Wu}$, et al., 2006, Wu, et al., 2016). A schematic workflow illustrating the steps about iTRAQ process applied in this study is shown in figure 1 .

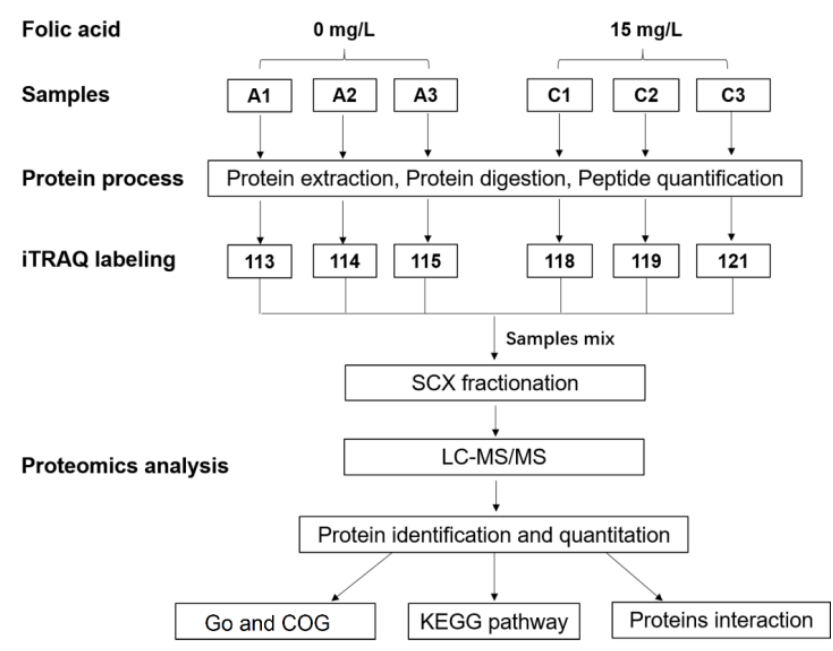

Figure 1. Experimental design and schematic diagram of proteomics analysis in the study.

Table 1. Primers of genes for RT-PCR analysis

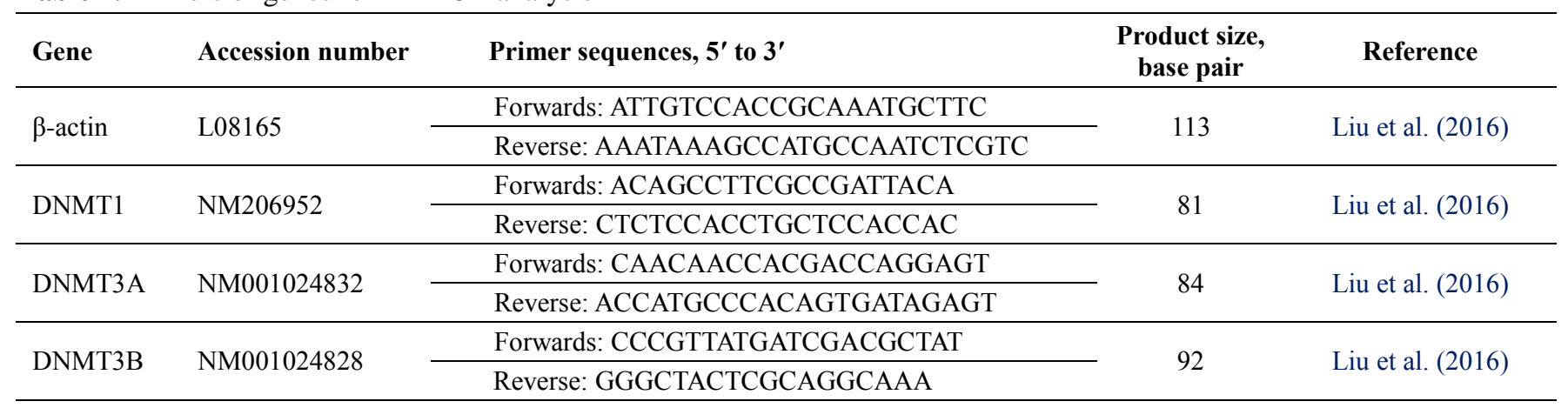

\section{Statistical analysis}

Experimental data on DNMTs expression, genomic $5 \mathrm{mC}$ level, folic acid, 5-methyl-THF and SAM contents in chicken hepatocytes were analyzed using $t$-test in SPSS 21.0 software (SPSS Inc., Chicago, IL, USA). The identification of differential expression proteins (DEPs) between two groups depended on the ratio of protein contents in folic acid free group to folic acid supplement group. The ratio $\geqslant 1.20$ or $\leqslant 0.83$ was regarded as differentially expressed proteins. In addition, a value of $\mathrm{P}$ $<0.05$ was considered to be statistically significant. 


\section{Ethics Committee Approval}

All the birds and experimental protocol in this study were approved by the Institution Animal Care and Use Committee of the Northwest A\&F University (protocol number NWAFAC1008).

\section{RESULTS}

\section{$5 \mathrm{mC}$ level and some metabolites content}

As shown in table 2, intracellular folic acid, 5-MeTHF and SAM contents were significantly higher in folic acid group when compared with folic acid free group $(\mathrm{P}<0.05)$. Whereas, there was no difference about genome $5 \mathrm{mC}$ levels between these two groups.

Table 2. Levels of genome $5 \mathrm{mC}$ and some metabolites in hepatocytes of layer chicks

\begin{tabular}{lcccc}
\hline Parameters & FA-free & FA-sup & SEM & P value \\
\hline $5 \mathrm{mC}(\%)$ & 1.00 & 0.81 & 0.106 & 0.096 \\
\hline FA $\left(\mathrm{ng} / 10^{6}\right.$ cell $)$ & 24.00 & $29.94^{*}$ & 0.430 & $<0.001$ \\
\hline 5-Me-THF $\left(\mathrm{ng} / 10^{6}\right)$ cell $)$ & 0.26 & $0.37^{*}$ & 0.024 & 0.002 \\
\hline SAM $\left(\mathrm{ng} / 10^{6}\right.$ cell $)$ & 1.69 & $1.96^{*}$ & 0.091 & 0.021 \\
\hline
\end{tabular}

Note: The symbol * showed difference significantly in statistics between folic acid free and supplement groups $(\mathrm{P}<0.05)$. SEM= Standard error; $\mathrm{FA}=$ folic acid; 5-Me-THF=5-methyl tetrahydrofolic acid; $\mathrm{SAM}=\mathrm{S}$ adenosylmethionine.

\section{mRNA expression of DNMTs}

As exhibited in figure $2,15 \mathrm{mg} / \mathrm{L}$ folic acid supplement didn't affect genes expression about DNA methyltransferases in comparison with those in folic acid free group.

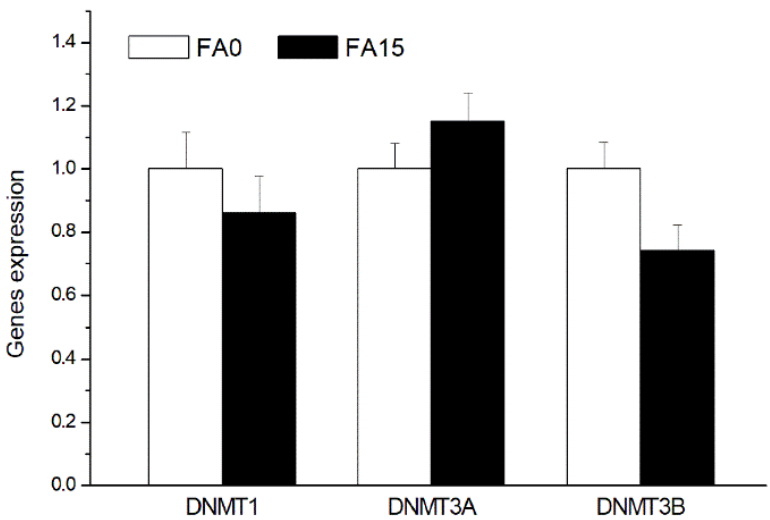

Figure 2. Gene expression of DNA Methyltransferases (DNMT1, DNMT3A, DNMT3B) in layer chicken hepatocytes between groups with folic acid free and supplement medium. Data were presented as means \pm $\operatorname{SEM}(n=6)$.

\section{Protein profiling}

Using the Mascot software, a total of 28725 unique peptides and 4660 proteins were identified. Among these proteins, 547 were between 0 to $20 \mathrm{kDa}, 2393$ between 20 to $60 \mathrm{kDa}, 965$ between 60 to $100 \mathrm{kDa}$ and 755 over 100 $\mathrm{kDa}$ (Figure 3A). 1405 proteins had one unique peptide, 670 had two, 667 had more than 11, and the left had 3-10 (Figure 3B). Because iTRAQ quantification indicated the amount of real fold change between groups to some extent, proteins with a fold-change $>1.2$ or $<0.83$ $(\mathrm{P}<0.05)$ were regarded as differential expressed proteins (DEPs). Based on this standard, 85 DEPs (35 downregulation and 50 up-regulation) were detected shown in table 3.

Table 3. Differential expression proteins between folic acid free and supplement groups

\begin{tabular}{|c|c|c|c|c|}
\hline Accession & Gene name & Protein name & $\begin{array}{l}{ }^{1} \text { Ratio } \\
\text { Sup/free }\end{array}$ & $P$ value \\
\hline \multicolumn{5}{|l|}{ Down-regulation } \\
\hline F1N804 & PLXNA1 & Plexin A1 & 0.529 & 0.012 \\
\hline F1NL76 & GAK & Cyclin G associated kinase & 0.641 & $<0.001$ \\
\hline Q8AWB6 & SLC35B 1 & Solute carrier family 35 member B1 & 0.675 & $<0.001$ \\
\hline R4GF71 & TMSB4X & AM-8-amino-7-oxononanoate aminotransferase & 0.707 & 0.002 \\
\hline E1B2Y2 & SLC7A3 & Cationic amino acid transporter-3 & 0.709 & 0.006 \\
\hline B5AIG4 & PNPLA2 & Adipose triglyceride lipase & 0.711 & 0.007 \\
\hline A0A1L1S044 & LOC420368 & Predicted GTPase & 0.711 & 0.038 \\
\hline F1P4D1 & SLC30A7 & Zinc transporter 7 & 0.746 & 0.031 \\
\hline A0A165FX80 & CATH1 & Cathelicidin-1 & 0.754 & 0.046 \\
\hline P12276 & FASN & Fatty acid synthase & 0.759 & 0.010 \\
\hline F1P3G3 & CHN1 & Chimerin 1 & 0.760 & 0.003 \\
\hline A0A2K6TZL8 & TSNAXIP1 & Translin associated factor $\mathrm{X}$ interacting protein 1 & 0.768 & 0.009 \\
\hline F1NDN6 & KRT12 & Keratin 12 & 0.768 & 0.001 \\
\hline H9L107 & KRT4 & Myosin heavy chain & 0.771 & 0.003 \\
\hline Q5ZJ43 & EXOC8 & Exocyst complex component 8 & 0.774 & 0.018 \\
\hline F1NGI6 & SGSH & $\mathrm{N}$-sulfoglucosamine sulfohydrolase & 0.779 & 0.011 \\
\hline $\mathrm{E} 1 \mathrm{C} 483$ & ACBD6 & Acyl-CoA-binding protein & 0.779 & 0.016 \\
\hline
\end{tabular}




\begin{tabular}{|c|c|c|c|c|}
\hline E1BS86 & AIG1 & Androgen induced 1 & 0.780 & 0.043 \\
\hline F1NQ90 & C11H19ORF12 & Mu-like prophage protein & 0.781 & 0.005 \\
\hline F1NNN3 & TCERG1L & Transcription elongation regulator 1 like & 0.783 & 0.022 \\
\hline F1NKU2 & MELK & Non-specific serine/threonine protein kinase & 0.783 & 0.017 \\
\hline P08286 & P08286 & Histone H1.10 & 0.787 & 0.011 \\
\hline E1BSR9 & RBX1 & Ring-box 1 & 0.787 & 0.049 \\
\hline F1NRK3 & RPP38 & Ribonuclease P/MRP subunit $\mathrm{p} 38$ & 0.792 & 0.013 \\
\hline F1NZ92 & DNAH3 & Dynein, heavy chain & 0.798 & 0.017 \\
\hline F1P4C2 & RIPK1 & Receptor interacting serine/threonine kinase 1 & 0.805 & 0.018 \\
\hline F1P2M3 & MTIF3 & Translation initiation factor 3 & 0.810 & 0.001 \\
\hline E1C4V2 & Gga.15193 & Zn-finger & 0.810 & 0.003 \\
\hline E1BSI3 & ENSGALG00000006435 & Ubiquitin-protein ligase & 0.814 & 0.036 \\
\hline F1NW64 & TPX2 & Microtubule nucleation factor & 0.817 & 0.012 \\
\hline E1C8Q1 & CEP164 & Centrosomal protein 164 & 0.821 & 0.005 \\
\hline E1BV18 & CAPSL & Calcyphosine like & 0.824 & $<0.001$ \\
\hline A0A0A0MQ61 & ENSGALG00000016325 & Glutathione S-transferase & 0.825 & 0.017 \\
\hline F1NWX7 & SEC61B & Transcription factor about chromatin remodeling & 0.826 & 0.023 \\
\hline R4GLJ6 & VHL & Phosphotransferase & 0.829 & 0.002 \\
\hline \multicolumn{5}{|l|}{ Up-regulation } \\
\hline F1NF85 & PEMT & Phosphatidylethanolamine N-methyltransferase & 1.200 & 0.019 \\
\hline E1C3M0 & USP45 & Ubiquitin specific peptidase 45 & 1.213 & 0.006 \\
\hline F1NPJ3 & CCDC127 & Translation initiation factor 2 & 1.215 & 0.036 \\
\hline E1BVP5 & ASPA & Aspartoacylase & 1.216 & 0.036 \\
\hline F1P2G6 & PIGT & Phosphatidylinositol glycan anchor biosynthesis & 1.220 & 0.036 \\
\hline R4GGH1 & ENSGALG00000028833 & NAD-dependent aldehyde dehydrogenases & 1.223 & 0.029 \\
\hline E1C0T3 & PDZRN3 & PDZ domain containing ring finger 3 & 1.225 & 0.013 \\
\hline F1NST0 & DHX58 & ERCC4-like helicases & 1.228 & 0.027 \\
\hline E6N1V0 & LAO & Amine oxidase & 1.229 & 0.008 \\
\hline P07031 & ACYP2 & Acylphosphatase-2 & 1.235 & 0.006 \\
\hline Q5ZLB2 & ARL6IP1 & Phosphoribosylaminoimidazole carboxylase & 1.240 & 0.018 \\
\hline E1C8Q2 & ETNPPL & 4-aminobutyrate aminotransferase & 1.243 & 0.001 \\
\hline F1ND79 & ZNF644 & Zn-finger & 1.254 & 0.013 \\
\hline Q5F366 & IDUA & Iduronidase & 1.259 & 0.020 \\
\hline F1NS64 & MFSD4B & Major facilitator superfamily domain containing 4B & 1.270 & 0.034 \\
\hline F1NK39 & ARID4B & histone trimethylation & 1.271 & 0.007 \\
\hline F1P3K7 & PCBD2 & Pterin-4-alpha-carbinolamine dehydratase 2 & 1.275 & 0.027 \\
\hline F1N8L2 & TECR & Very-long-chain enoyl-CoA reductase & 1.285 & 0.034 \\
\hline F1NJK5 & RRP7A & Ribosomal RNA processing 7 homolog A & 1.295 & 0.006 \\
\hline Q5ZJC0 & RCJMB04 & Uncharacterized protein & 1.303 & 0.022 \\
\hline E1BUS8 & CRYZL1 & Quinone oxidoreductase-like protein 1 & 1.317 & 0.011 \\
\hline Q9W7G2 & SALL3 & Spalt protein & 1.355 & 0.017 \\
\hline F1P054 & CYP1A5 & Cytochrome P450 & 1.363 & 0.001 \\
\hline E1C7X0 & VRK2 & Serine/threonine protein kinase & 1.367 & 0.018 \\
\hline F1NB56 & PDE4D & Phosphodiesterase & 1.368 & 0.019 \\
\hline B8XA33 & ADAM23 & Disintegrin and metalloprotease 23 & 1.374 & 0.016 \\
\hline F1P3X6 & YTHDF2 & Membrane proteins & 1.377 & 0.001 \\
\hline R4GG24 & AKR1B1L & Aldo/keto reductases & 1.380 & 0.041 \\
\hline E1BZD6 & RIPK4 & Ankyrin repeat & 1.382 & 0.013 \\
\hline F1NWY7 & MRPL42 & Mitochondrial ribosomal protein L42 & 1.391 & 0.010 \\
\hline Q5ZL23 & APBB1IP & Protein-binding family interacting protein & 1.395 & 0.014 \\
\hline Q5ZJ36 & PLK1 & Serine/threonine-protein kinase PLK & 1.413 & 0.008 \\
\hline F1P586 & SFSWAP & Splicing factor SWAP & 1.428 & 0.002 \\
\hline O73884 & PHOSPHO1 & Phosphoethanolamine/phosphocholine phosphatase & 1.429 & 0.042 \\
\hline Q5ZLE1 & PRPF4B & Permeases & 1.438 & 0.045 \\
\hline G4XJS0 & TLR1LB & Toll-like receptor 1 type 2 & 1.447 & 0.004 \\
\hline Q5ZHK9 & LLPH & Protein LLP homolog & 1.456 & 0.015 \\
\hline F1NW34 & KDM8 & Lysine demethylase 8 & 1.459 & 0.024 \\
\hline E1C4M9 & SLC43A2 & Solute carrier family 43 member 2 & 1.473 & 0.027 \\
\hline F1P5K8 & APTX & Aprataxin & 1.575 & 0.009 \\
\hline F1NI14 & TXLNG & Taxilin gamma & 1.594 & 0.011 \\
\hline E1C6E5 & TSPAN3 & Tetraspanin & 1.630 & 0.036 \\
\hline E1C6D5 & KDM4A & PHD zinc finger-containing protein & 1.910 & 0.028 \\
\hline P28568 & SLC2A3 & Solute carrier family 2 & 2.119 & 0.020 \\
\hline Q5ZIB9 & PRMT7 & Protein arginine N-methyltransferase 7 & 2.169 & 0.020 \\
\hline R4GJY5 & FAM108A1 & Protein ABHD17B & 2.179 & 0.049 \\
\hline Q5F4A8 & AK6 & Adenylate kinase isoenzyme 6 & 2.246 & 0.041 \\
\hline G8HUH5 & BACT & Beta-actin (Fragment) & 2.331 & 0.006 \\
\hline Q5ZK96 & BTBD9 & BTB domain containing 9 & 2.518 & 0.045 \\
\hline Q5F3Q0 & NUP205 & Asp-tRNAAsn/Glu-tRNAGln amidotransferase & 3.167 & 0.014 \\
\hline
\end{tabular}

${ }^{1}$ Ratio sup/free $=$ Protein expression in folic acid supplement group $/$ that in folic acid free group . 


\section{Classification of DEPs}

Of the 85 DEPs, 79 DEPs could be assigned to 21 categories using the COG database. As shown in figure 4, the largest group was general function prediction only (26.6\%) followed by amino acid transport and metabolism $(10.1 \%)$, replication, recombination and repair $(10.1 \%)$. carbohydrate transport and metabolism (7.6\%), transcription (7.6\%), posttranslational modification, protein turnover, chaperones (6.3\%), and signal transduction mechanisms (6.3\%). Further, GO classification analysis of DEPs was performed. The number for significant enriched biological process, cell component and molecular function is 162,29 and 75 respectively (data not shown). In terms of GO term distributions in the second level as presented in figure 5, for biological processes, more than $60 \%$ of the notable proteins were respectively related to regulation of cellular process, single-organism process, and metabolic process; for cell component, about $68 \%, 57 \%$ and $35 \%$ were correlated with cell, organelle and membrane respectively; for molecular function, about 59\% and 50\% were respectively associated with binding and catalytic activity.

To characterize the functional consequences of DEPs associated with folic acid intervention in chicken primary hepatocytes, KEGG pathway mapping based on DEPs were also carried out and demonstrated in figure 6. Results indicated that folic acid could significantly affect metabolism of xenobiotics by cytochrome P450, drug metabolism- cytochrome P450, retinol metabolism, steroid hormone biosynthesis, pyruvate metabolism, tryptophan metabolism and glutathione metabolism. It was worth mentioning that some proteins such as ENSGALG00000016325, CYP1A5 and ACYP2 were involved in these pathways. ENSGALG00000016325 could code glutathione S-transferase which was downregulated in $15 \mathrm{mg} / \mathrm{L}$ folic acid group, while CYP1A5 and ACYP2 were up-regulated when compared with the no folic acid group which coded cytochrome P450 and acylphosphatase proteins respectively.

\section{Proteins interaction analysis}

The protein-protein interaction networks were performed by the web-tool STRING 10.5 (https://stringdb.org/cgi/input.pl). The DEPs interactions were shown in figure 7 , in which the stronger associations are represented by thicker lines. The results showed that some functional modules were clustered in the network and formed tight connections with DEPs in chicken primary hepatocytes between folic acid free and supplement groups.
Disconnected nodes in the network were hided. The functional modules were mainly involved in cell cycle (SKP1, RBX1, SKP2, CDC27, CDC20, MAD2L1, CUL1, BUB1B, PLK1, BUB1 and CCNB2), ubiquitin mediated proteolysis (CUL4A, CUL2, TCEB1, RBX1, SKP1, FBXW7, SKP2, CDC27, VHL, CUL1 and CDC20), protein export (SEC63, SEC61A1, SEC61B, SEC61G and SEC61A2), protein processing in endoplasmic reticulum (SEC63, SEC61A1, SEC61B, SEC61G, SEC61A2, SKP1, RBX1 and CUL1), phagosome (SEC61A1, SEC61B, SEC61G, SEC61A2, and ACTB), lysosome (IDUA, GALNS, CLTC and CLTCL1), ribosome biogenesis in eukaryotes (LOC425215, RRP7A and RPP38), TGF beta signaling pathway (SKP1, RBX1 and CUL1) and fatty acid biosynthesis (FASN and ENSGALG00000005439).
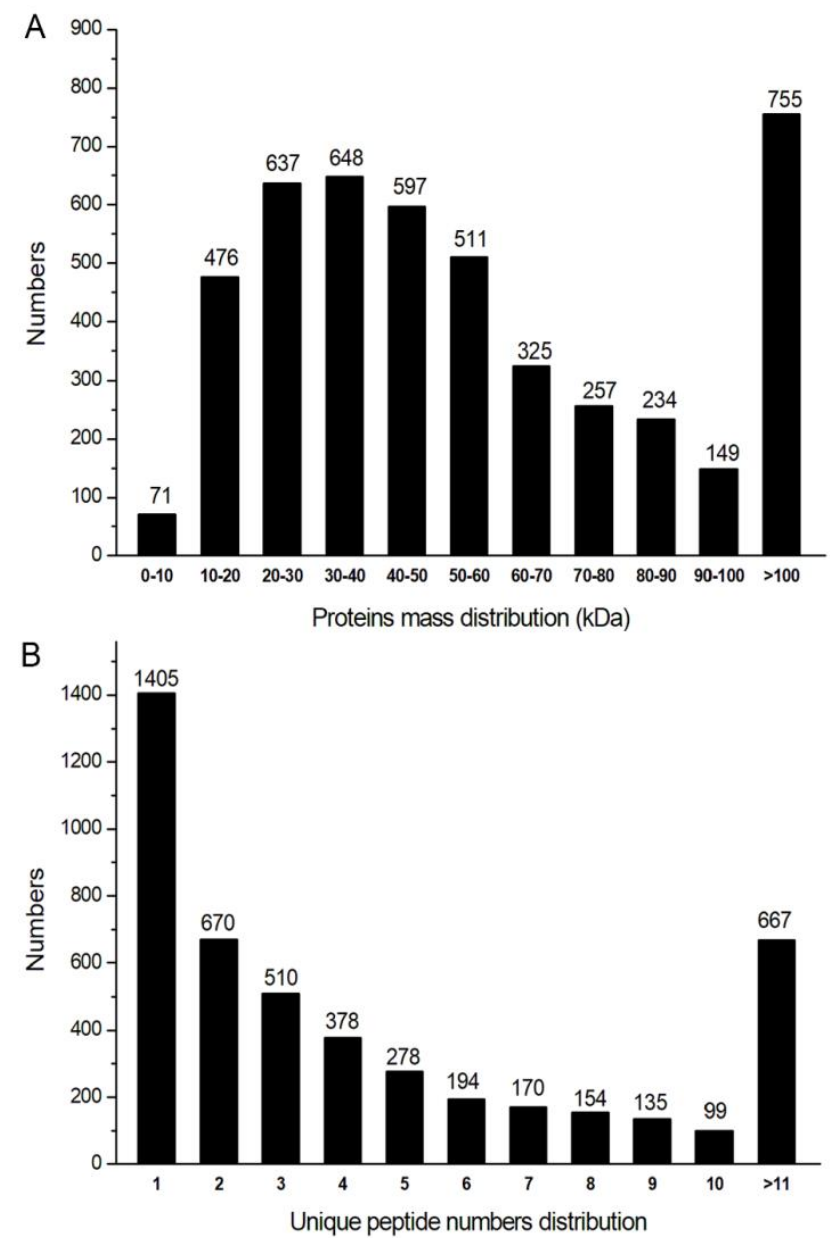

Figure 3. Basic information of iTRAQ identification. A: Different molecular weights distribution of proteins identified among samples. B: The number of unique peptides that identified proteins in the current study. 


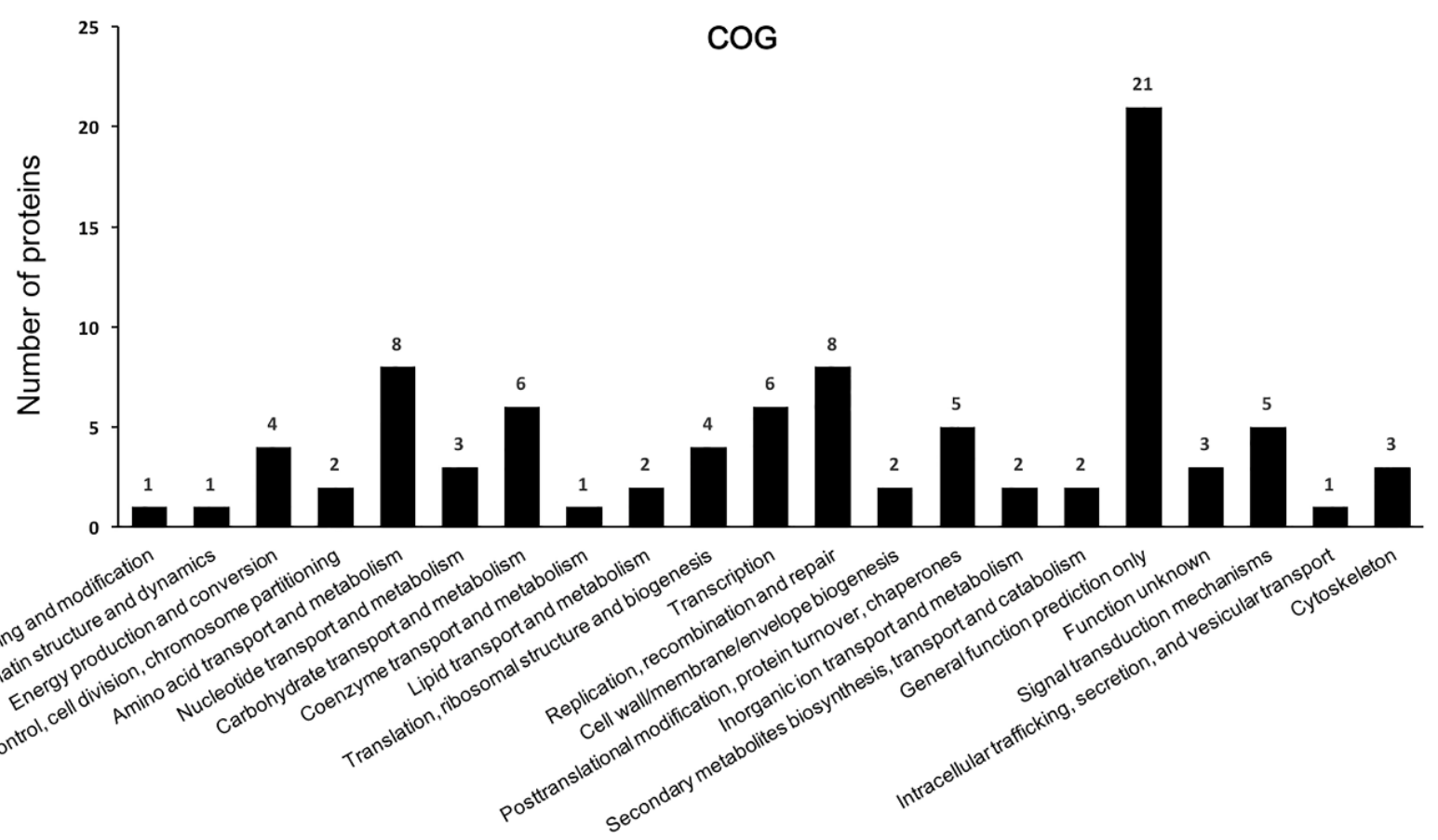

Figure 4. Clusters of Orthologous Groups (COG) of proteins classification of DEPs between folic acid free and supplement groups. The $\mathrm{Y}$-axis is the numbers of DEPs annotated to the category.

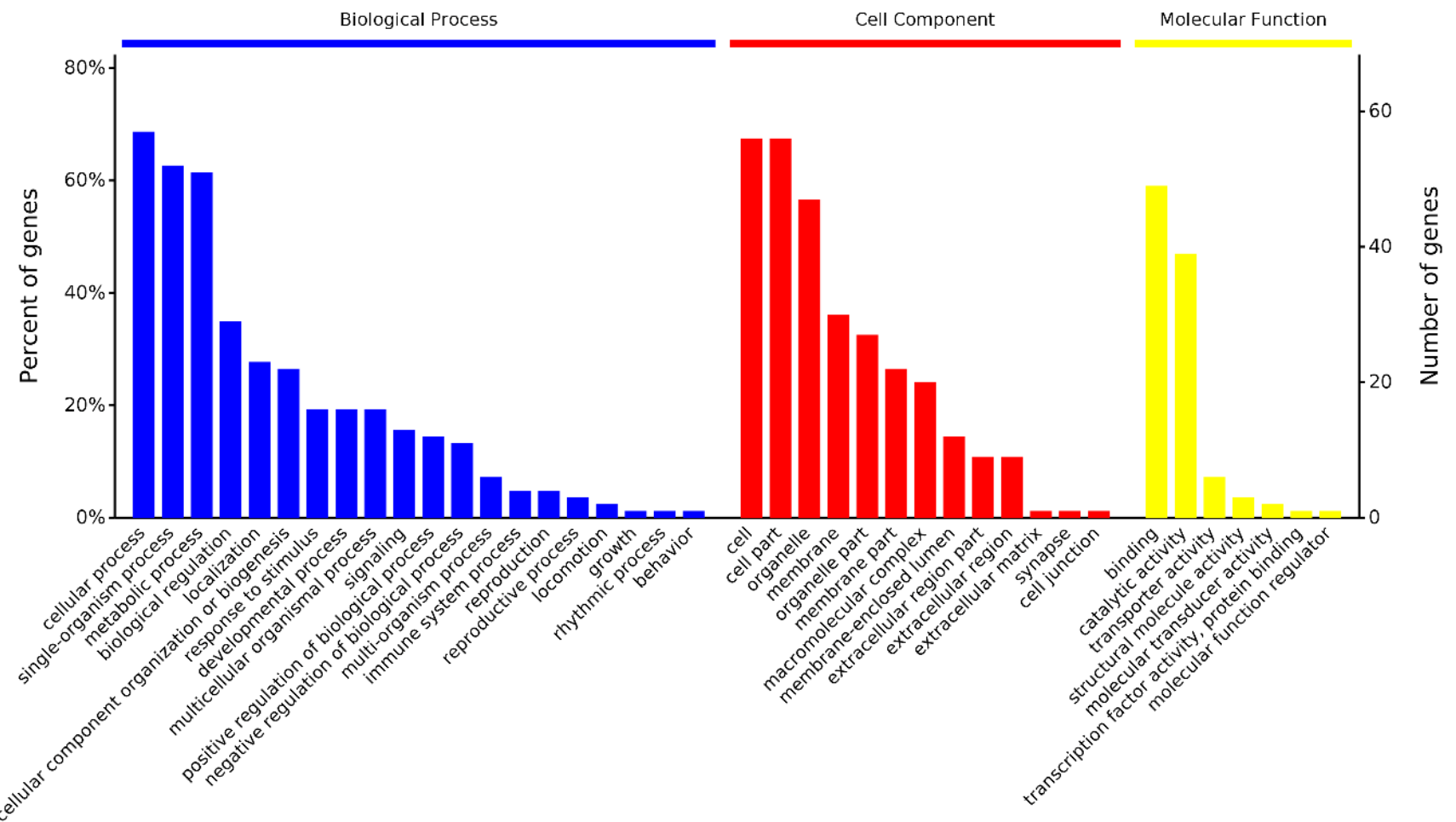

Figure 5. Functional classification of differential proteins by Gene Ontology analysis including biological process, cellular component, and molecular function. All data are presented based on GO second-level terms. The Y-axis is on behalf of the numbers of DEPs annotated to the corresponding category. 


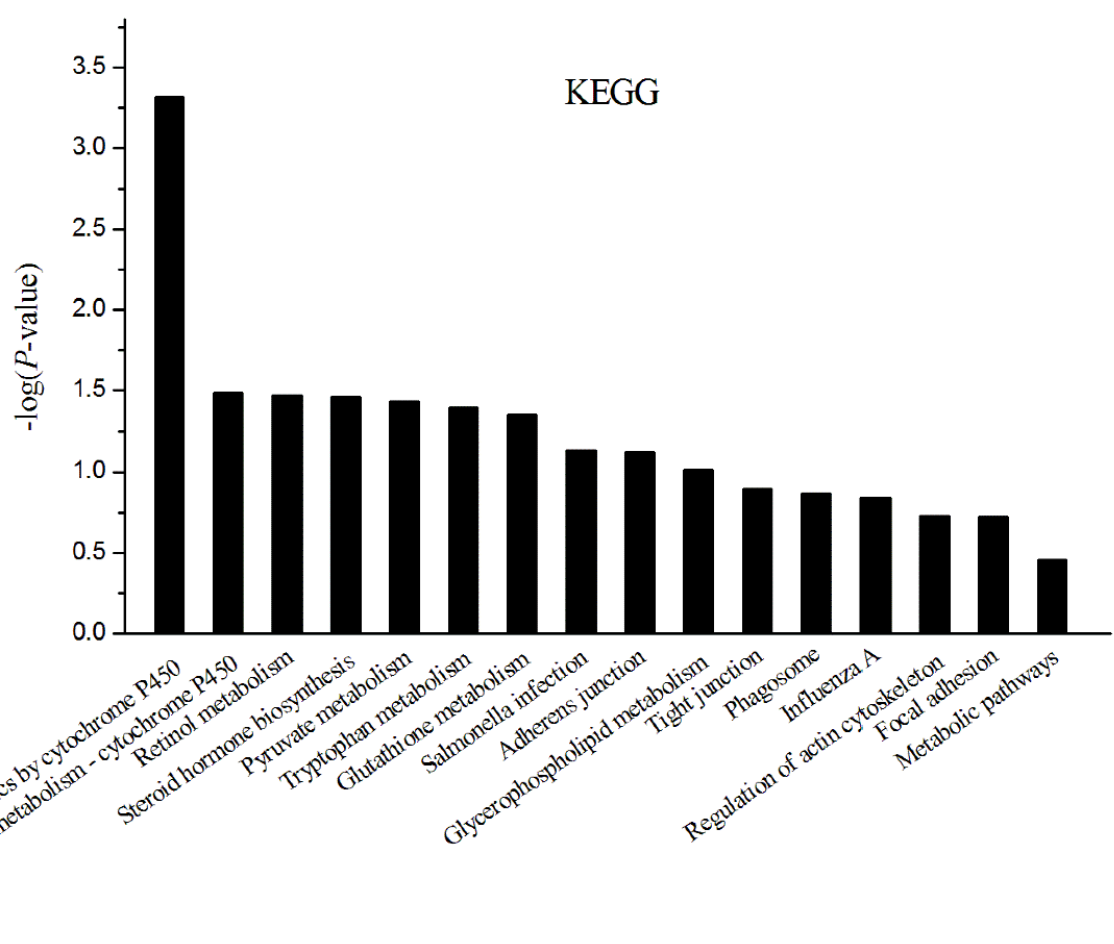

Figure 6. Distribution of enriched KEGG pathway according to DEPs between folic acid free and supplement groups.

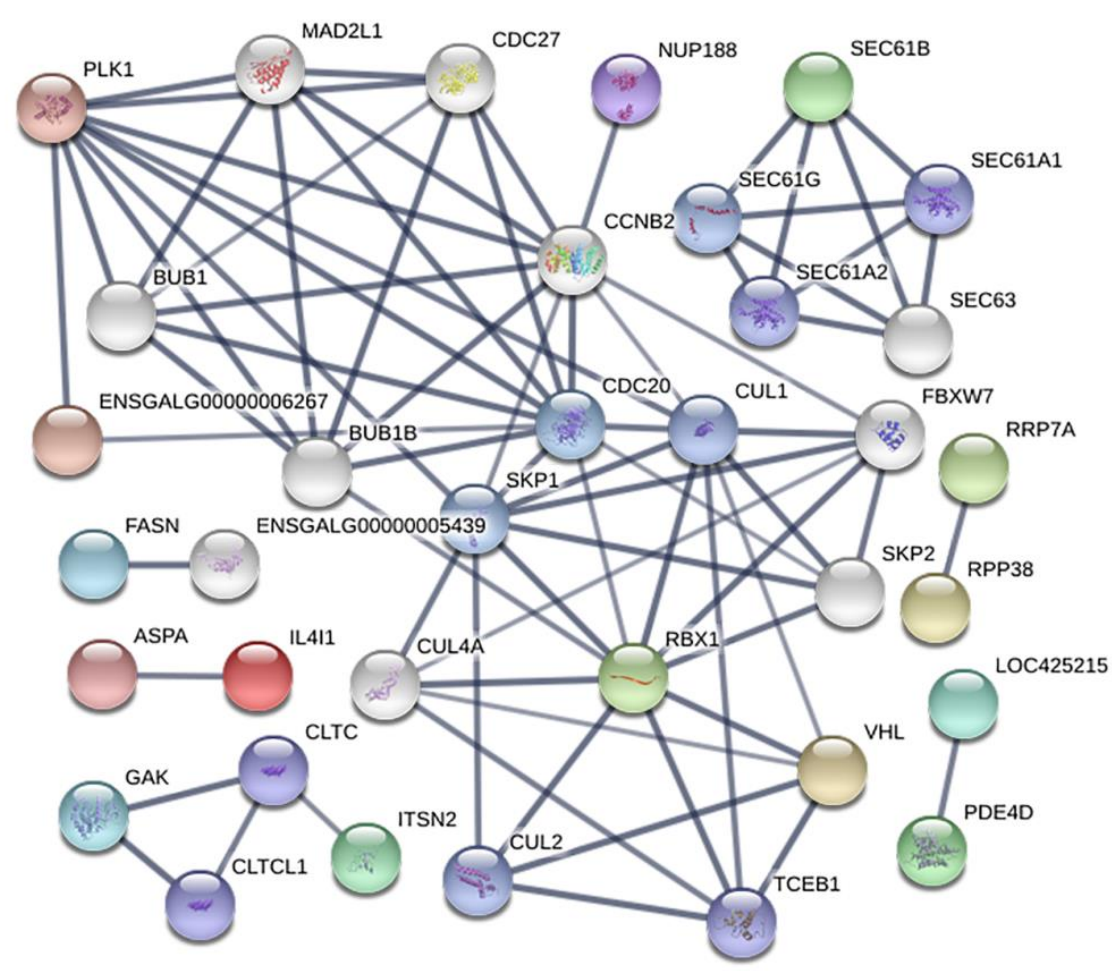

Figure 7. Interaction network analysis of DEPs using STRING software (http://string-db.org). In this network, nodes are proteins; lines represent functional associations between proteins. The resulting networks were constructed with confidence scores higher than 0.7. The gray lines between bodes represent functional associations between proteins and the thickness of the lines represents the level of confidence in association reported. 


\section{DISCUSSION}

In the current study, chicken primary hepatocytes are used as the model to explore folic acid metabolism regulation function through deprivation and supplementation ways. As we all known, folic acid was commonly recognized due to its significance for the development of neurological systems in newborns. Many study have stated that there existed negative correlations between dietary or plasma folic acid and the occurrence rate of some diseases (Sie et al., 2011, Chen et al., 2014, Molloy et al., 2017). But the causal mechanisms that define the role of folic acid in these complex diseases are not established. It's generally accepted that folic acid-mediated 1-carbon metabolism could affect genes expression by DNA methylation and chromatin structure, thereby disturbing metabolic pathways about pathologies (Stover, 2009). Previous study pointed out that folic acid could slow down the aggressiveness of glioma by increasing methylation levels of DNA repeats element and genes related to apoptosis and proliferation (Hervouet et al., 2009). It was reported that low folate intake could result in genomic DNA hypomethylation and improve the risk of colorectal neoplasia, and daily supplementation with $400 \mathrm{mg} /$ day folic acid for 10 weeks resulted in a marginal increase in leucocyte DNA methylation and rectal mucosa DNA methylation in patients with colorectal adenoma (Pufulete et al., 2005).

Considering the role of folic acid in DNA methylation and the fact that DNA methylation is critical to normal genome regulation and development (Crider et al., 2012), we examined genomic 5-methylcytosine (5mC) contents in hepatocytes with folic acid free and supplementation medium. Surprisingly, folic acid didn't increase DNA methylation level in the folic acid addition group. DNA methylation is catalyzed by DNA methyltransferases (DNMTs). DNMT1 is a maintenance methyltransferase and responsible for restoring the methylated status of newly synthesized daughter strands; DNMT3a and DNMT3b are de novo methyltransferases (Li et al., 2016). Consistently, these DNMTs expression were also not affected by folic acid supplementation in the current study. However, intracellular folic acid, 5-Me-THF and SAM concentrations were higher in culture medium with folic acid supplemented when compared with folic acid free group. These results may be illogicality taken together, but the relationship between folic acid and DNA methylation is complex. DNA methylation also involved in the participation of other substances such as choline, betaine and other B vitamins (Niculescu and Zeisel, 2002). On the other hand, SAM could inhibit MTHFR activity, which provides 5 -Me-THF by catalyzing a unidirectional reaction (Smith et al., 2013). But other review also suggested that there was no correlation between global DNA methylation and folate status (Crider et al., 2012).

In addition, there was no difference about cell viability, albumin and lactic dehydrogenase concentration in culture medium between folic acid free and addition groups (data not shown), which suggested that the dosage of folic acid used in the study was reasonable and nontoxic for cells growth. Hence, proteomic analysis was further employed to assess folic acid metabolism regulation function in primary chicken hepatocytes. We found folic acid changed some metabolic pathways enriched by 85 DEPs including cytochrome P450 metabolism, retinol metabolism, steroid hormone biosynthesis, pyruvate metabolism, tryptophan metabolism and glutathione metabolism. Cytochrome P450 was reported to be involved in oxidation-reduction reactions (Meunier, et al., 2004), and up-regulated in the current study indicating that folic acid improved antioxidant ability. ENSGALG00000016325 which coded glutathione S-transferase (GSTs) was also contained in the pathway of cytochrome P450 metabolism, and was down-regulated in folic acid addition group. GSTs are the ubiquitous enzymes that play a key role in cellular detoxification (Jain et al., 2010), and its lower protein abundance suggested that folic acid seemed to be protective for hepatocytes. Folic acid, as an antioxidant (Gliszczynskaswiglo, 2007), has good therapeutic effects on hypoxia-induced inflammatory response by decreasing ROS activity (Ma et al., 2018).

Besides, retinol metabolism, steroid hormone biosynthesis, pyruvate metabolism, and tryptophan metabolism were also enriched. These could be contained amino acid and carbohydrate metabolism as COG analysis that amino acid or carbohydrate transport and metabolism were clustered in relative high proportion. However, how does folic acid affect these metabolism change? It is interesting to note that arginine $\mathrm{N}$-methyltransferase 7 (PRMT7) and ARID4B were up-regulated proteins by folic acid addition based on proteomics though no evidence was found about DNA methylation. PRMT7 has been implicated in roles of transcriptional regulation, DNA damage repair, RNA splicing, cell differentiation, metastasis and epigenetic regulation by transferring methyl groups to arginine residues on protein substrates (Feng et al., 2013). Biological process analysis of GO has 
suggested that ARID4B was associated with histone H3K9 and H4K20 trimethylation which were all related to nucleosome and chromatin structure (Xu et al., 2008, Hahn et al., 2011). These results indicated that folic acid might take part in metabolism regulation by histone methylation which contributed to transcription and posttranscriptional modification. And posttranslational modification, protein turnover, chaperones and transcription were gathered by COG analysis based on DEPs. Li et al. (2016a) has reported that folic acid increased H3K9 methylation of IL-6 promoter. Therefore, we speculated that folic acid might regulate hepatocellular metabolism via the histone methylation manner rather than DNA methylation in the present study.

\section{CONCLUSION}

In conclusion, the present proteomic analysis found 85 differential expressed proteins in primary chicken hepatocytes with folic acid free and supplementation medium. The pathways of those altered proteins are related to amino acid and carbohydrate metabolism, and oxidation resistance. Folic acid regulated these metabolisms more likely by histone methylation rather than DNA methylation. These results indicated that proteomics with bioinformatics analysis is a good starting point for understanding regulation function of some substances. A deep and broad understanding of the DEPs identified is ongoing to make clear their specific role. Our findings might provide comprehensive protein expression information that can facilitate the understanding of folic acid regulation function in hepatic metabolism.

\section{DECLARATIONS}

\section{Competing interests} interests.

The authors declare that they have no competing

\section{Author`s contributions}

XJY and YLL designed the research; JFZ, FYW, JHZ and YLL performed the research and analysed the data; YLL wrote the manuscript; XY and XJY have taken part in the revision of the manuscript. All authors read and approved the final version of the manuscript.

\section{Acknowledgments}

This work was funded by the National Science Foundation of China (No. 31972529), the Program for Shaanxi Science \& Technology (2018ZDCXL-NY-0201, 2018ZDXM-NY-051), and the Program for Yangling Agricultural High-tech Industries Demonstration Zone
(2018CXY-10). This work was also supported in part by the scholarship from China Scholarship Council under the Grant CSC201906300069.

\section{REFERENCES}

Allah ESHA and Badary DM (2017). Folic acid protects against lead acetate-induced hepatotoxicity by decreasing nf- $\kappa b$, il-1 $\beta$ production and lipid peroxidation mediataed cell injury. $\begin{array}{llll}\text { Pathophysiology, } & 24 & \text { (1): } & 39-44 .\end{array}$ https://doi.org/10.1016/j.pathophys.2017.02.002

Cao Z, Meng B, Fan R, Liu M, Gao M, Xing Z and Luan X (2018). Comparative proteomic analysis of ovaries from huoyan geese between pre-laying and laying periods using an itraq-based approach. Poultry Science, 1: 1-13. DOI: https://doi.org/10.3382/ps/pey029

Chen P, Li C, Li X, Li J, Chu R and Wang H (2014). Higher dietary folate intake reduces the breast cancer risk: A systematic review and meta-analysis. British Journal of Cancer, 110 (9): 2327-2338. DOI: https://doi.org/10.1038/bjc.2014.155

Chen X, Che C, Korolchuk VI, Gan F, Pan C and Huang K (2017). Selenomethionine alleviates afb1-induced damage in primary chicken hepatocytes by inhibiting cyp450 1a5 expression via upregulated selw expression. Journal of Agricultural and Food Chemistry, $65 \quad$ (12): 2495-2502. DOI: https://doi.org/10.1021/acs.jafc.6b05308

Crider KS, Yang TP, Berry RJ and Bailey LB (2012). Folate and DNA methylation: A review of molecular mechanisms and the evidence for folate's role. Advances in Nutrition: An International Review Journal, 3 (1): 21-38. DOI: https://doi.org/10.3945/an.111.000992

Cui S, Li W, Lv X, Wang P, Huang G and Gao Y (2017). Folic acid attenuates homocysteine and enhances antioxidative capacity in atherosclerotic rats. Applied Physiology Nutrition \& Metabolism, 42: 1015-1022. DOI: https://doi.org/10.1139/apnm-2017-0158

Dong WT, Xiao LF, Hu JJ, Zhao XX, Liu JX and Zhang Y (2017). ITRAQ proteomic analysis of the interactions between bombyx mori nuclear polyhedrosis virus and silkworm. Journal of Proteomics, 166: 138-145. DOI: https://doi.org/10.1016/j.jprot.2017.07.013

Du M, Liu X, Liu X, Yin X, Han S, Song Q and An S (2015). Glycerol3-phosphate O- acyltransferase is required for PBAN-induced sex pheromone biosynthesis in Bombyxmori. Scientific Reports, 5: 8110-8119. DOI: https://doi.org/10.1038/srep08110

Feng Y, Maity R, Whitelegge JP, Hadjikyriacou A, Li Z, Zurita-Lopez C, Al-Hadi Q, Clark A, Bedford M, Masson J and Clarke S (2013). Mammalian protein arginine methyltransferase 7 (prmt7) specifically targets rxr sites in lysine- and arginine-rich regions. Journal of Biological Chemistry, 288 (52): 37010-37025. DOI: 10.1074/jbc.M113.525345

Gliszczynskaswiglo A (2007). Folates as antioxidants. Food Chemistry, $101 \quad(4)$ : 1480-1483. DOI https://doi.org/10.1016/j.foodchem.2006.04.022

Hahn MA, Wu X, Li AX, Hahn T and Pfeifer GP (2011). Relationship between gene body DNA methylation and intragenic h3k9me3 and h3k36me3 chromatin marks. Plos One, 6 (4): e18844. DOI: https://doi.org/10.1371/journal.pone.0018844

Hervouet E, Debien E, Campion L, Charbord J, Menanteau J, Vallette F and Cartrone P (2009). Folate supplementation limits the aggressiveness of glioma via the remethylation of DNA repeats element and genes governing apoptosis and proliferation. Clinical Cancer Research, 15 (10): 3519-3529. DOI: 10.1158/1078-0432.

Hou DX, Kunitake T, Kusuda J and Fujii M (2001). Primary culture of chicken hepatocytes as an in vitro model for determining the influence of dioxin. Bioscience, Biotechnology, and Biochemistry, 65 (1): 218-221. DOI: https://doi.org/10.1271/bbb.65.218 
Jain M, Ghanashyam C and Bhattacharjee A (2010). Comprehensive expression analysis suggests overlapping and specific roles of rice glutathione s-transferase genes during development and stress responses. BMC Genomics, 11 (1): 73-90. DOI: https://doi.org/10.1186/1471-2164-11-73

Lambrot R, Xu C, Saintphar S, Chountalos G, Cohen T, Paquet M, Suderman M, Hallett M and Kimmins S (2013). Low paternal dietary folate alters the mouse sperm epigenome and is associated with negative pregnancy outcomes. Nature Communications, 4 (4): 2889-2903. DOI: https://doi.org/10.1038/ncomms3889

Li S, Zhi L, Liu Y, Shen J, Liu L, Yao J and Yang X (2016a). Effect of in ovo feeding of folic acid on the folate metabolism, immune function and epigenetic modification of immune effector molecules of broiler. British Journal of Nutrition, 115 (03): 411421. DOI: https://doi.org/10.1017/S0007114515004511

Li S, Zhu Y, Zhi L, Han X, Shen J, Liu Y, Yao J and Yang X (2016b). DNA methylation variation trends during the embryonic development of chicken. Plos One, 11 (7): e0159230. DOI: https://doi.org/10.1371/journal.pone.0159230

Liu Y, Guo W, Pu Z, Li X, Lei X, Yao J and Yang X (2016). Developmental changes of insulin-like growth factors in the liver and muscle of chick embryos. Poultry Science, 95 (6): 1396-1402. DOI: https://doi.org/10.3382/ps/pew043

Liu Y, Wu S, Sun W, Chen S, Yang X, and Yang X (2018). Variation in proteomics and metabolomics of chicken hepatocytes exposed to medium with or without folic acid. Journal of Cellular Biochemistry, $\quad 119$ : 6113-6124. DOI: https://doi.org/10.1002/jcb. 26810

Livak KJ and Schmittgen TD (2001). Analysis of relative gene expression data using real-time quantitative PCR and the 2 (-Delta Delta C(T)) method. Methods, 25 (4): 402-408. DOI: https://doi.org/10.1006/meth.2001.1262

Lucock M (2000). Folic acid: Nutritional biochemistry, molecular biology, and role in disease processes. Molecular Genetics \& $\begin{array}{llll}\text { Metabolism, } & 71 & (1-2): & 121-138\end{array}$ https://doi.org/10.1006/mgme.2000.3027

Ma J, Zhen X, Huang X and Jiang X (2018). Folic acid supplementation repressed hypoxia-induced inflammatory response via ros and jak2/stat3 pathway in human pro-myelomonocytic cells. Nutrition Research, 53: 40-50. DOI: https://doi.org/10.1016/j.nutres.2018.03.007

Meunier B, de Visser SP and Shaik S (2004). Mechanism of oxidation reactions catalyzed by cytochrome p450 enzymes. Chemical

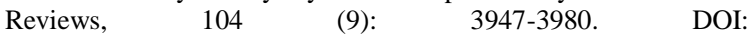
https://doi.org/10.1021/cr020443g

Molloy AM, Pangilinan F and Brody LC (2017). Genetic risk factors for folate responsive neural tube defects. Annual Review of Nutrition, 37 (1): 269-291. DOI: https://doi.org/10.1146/annurev-nutr071714-034235

Nazki FH, Sameer AS and Ganaie BA (2014). Folate: Metabolism, genes, polymorphisms and the associated diseases. Gene, 533 (1): 11-20. DOI: https://doi.org/10.1016/j.gene.2013.09.063
Niculescu MD and Zeisel SH (2002). Diet, methyl donors and DNA methylation: Interactions between dietary folate, methionine and choline. Journal of Nutrition, 132 (8 Suppl): 2333s-2335s. DOI: https://doi.org/10.1093/jn/132.8.2333S

Pufulete M, Al-Ghnaniem R, Khushal A, Appleby P, Harris N, Gout S, Emery PW and Sanders TAB (2005). Effect of folic acid supplementation on genomic DNA methylation in patients with colorectal adenoma. Gut, 54 (5): 648-653. DOI: 10.1136/gut.2004.054718

Sie KK, Medline A, Van Weel J, Sohn K-J, Choi S-W, Croxford R, and Kim Y (2011). Effect of maternal and postweaning folic acid supplementation on colorectal cancer risk in the offspring. Gut, 60 (12): 1687-1694. DOI: http://dx.doi.org/10.1136/gut.2011.238782

Smith DE, Hornstra JM, Kok RM, Blom HJ and Smulders YM (2013). Folic acid supplementation does not reduce intracellular homocysteine, and may disturb intracellular one-carbon metabolism. Clinical Chemistry and Laboratory Medicine, 51 (8): 1643-1650. DOI: https://doi.org/10.1515/cclm-2012-0694

Stover PJ (2009). One-carbon metabolism-genome interactions in folateassociated pathologies. Journal of Nutrition, 139 (12): 2402-2405. DOI: https://doi.org/10.3945/jn.109.113670

Wang L, Liang W, Xing J, Tan F, Chen Y, Huang L, Chen C and Chen W (2013). Dynamics of chloroplast proteome in salt-stressed mangrove kandelia candel (1.) druce. Journal of Proteome Research, $12 \quad$ (11): $5124-5136 . \quad$ DOI: https://doi.org/10.1021/pr4006469

Wu J, Mao X, Cai T, Luo J and Wei L (2006). Kobas server: A webbased platform for automated annotation and pathway identification. Nucleic Acids Research, 34 (Web Server issue): 720-724. DOI: https://doi.org/10.1093/nar/gk1167

Wu Y, Tang J, Zhou C, Zhao L, Chen J, Zeng L, Rao C, Shi H, Liao L and Liang $Z$ (2016). Quantitative proteomics analysis of the liver reveals immune regulation and lipid metabolism dysregulation in a mouse model of depression. Behavioural Brain Research, 311: 330-339. DOI: https://doi.org/10.1016/j.bbr.2016.05.057

Xu L, Simon MD, Chodaparambil JV, Hansen JC, Shokat KM, and Luger K (2008). The effect of h3k79 dimethylation and h4k20 trimethylation on nucleosome and chromatin structure. Nature Structural \& Molecular Biology, 15 (10): 1122-1124. DOI: https://doi.org/10.1038/nsmb.1489

Xu X, Diao J, Wang X, Dang Z, Zhang P, Li Y and Zhou Z (2012). Enantioselective metabolism and cytotoxicity of the chiral herbicide ethofumesate in rat and chicken hepatocytes. Pesticide Biochemistry and Physiology, 103 (1): 62-67. DOI: https://doi.org/10.1016/j.pestbp.2012.03.005

Yu X, Liu R, Zhao G, Zheng M, Chen J and Wen J (2014). Folate supplementation modifies ccaat/enhancer-binding protein alpha methylation to mediate differentiation of preadipocytes in chickens. Poultry Science, 93 (10): 2596-2603. DOI: https://doi.org/10.3382/ps.2014-04027 